\title{
Absorption and phase imaging with
}

\section{synchrotron radiation}

\author{
Peter Cloetens ${ }^{1}$, Elodie Boller ${ }^{1}$, Wolfgang Ludwig' ${ }^{1}$ José Baruchel ${ }^{1}$ and Michel Schlenker ${ }^{2}$ \\ ${ }^{I}$ European Synchrotron Radiation Facility, B.P. 220, F-38043 Grenoble, France \\ ${ }^{2}$ Laboratoire Louis Néel du CNRS, B.P. 166, F-38042 Grenoble, France
}

A 11 users agree on two essential virtues of synchrotron radiation: its high intensity in the $\mathrm{X}$-ray range, and its continuous spectrum. The new, third generation, sources have another, at first sight less spectacular feature: the small divergence of the beam as seen from the sample. This characteristic is due to the very small cross-sectional area of the electron beam that acts as the source of radiation, and to the large source - sample distance. All three of these qualities lead to novel possibilities, among others in X-ray imaging. We will discuss some of the approaches developed in hard X-ray synchrotron radiation imaging, and some of its applications in quite diverse fields.

Synchrotron radiation refers to the electromagnetic radiation emitted by ultrarelativistic electrons (energies of several GeV), circulating in storage rings, at those parts of the rings where they are accelerated by a magnetic field. This can be uniform over a part of the trajectory in the bending magnets, or spatially oscillating in the "insertion devices". The spectrum of the light thus produced extends from the infra red into the X-ray range, the latter part being to most users the more valuable one. Emission is strongly concentrated in the forward direction with respect to the velocity of the emitting electrons, the characteristic angular opening being $m c^{2} / E$, with $E$ the energy of the electrons, $m$ their rest mass. Three machines in the world belong to the category of third generation, high energy sources : the European Synchrotron Radiation Facility (ESRF) in Grenoble, France, at $6 \mathrm{GeV}$; the Advanced Photon Source in Argonne, IL, USA at $7 \mathrm{Gev}$; and SPRING-8 in Japan, at $8 \mathrm{GeV}$. They are characterised by the thinness of the electron beam that produces the radiation (source dimensions $<0.1 \mathrm{~mm}$ ), and by the provision, in between the bending magnets, of many straight sections. These allow the positioning of insertion devices, viz. wigglers or undulators, which can provide each experiment with the best suited beam.

Imaging is normally associated, in our minds, with lenses. Unlike visible light or electrons, efficient lenses are not (yet?) available for hard X-rays, essentially because they interact weakly with matter, resulting in a refractive index very close (to within $10^{-5}$ or $10^{-6}$ ) to unity. Nevertheless $\mathrm{X}$-ray imaging plays an immense role. Radiographs of hands of attendants made at the lectures on $\mathrm{X}$ rays in 1896 are the historical banner of X-rays, while each of us benefited from medical radiography and enjoys the leak-tightness which industrial radiography controls in pipelines. X-ray tomography is used on a routine basis, under the name of computed axial tomography (CAT) or medical scanner, to visualise virtual cuts through human anatomy, obtained from attenuation measurements performed under different viewing angles. In radiography (two-dimensional images) as well as in tomography (three-dimensional exploration), only contrast associated with local variations in X-ray absorption was, until recently, considered. On the other hand, a different approach, based on Bragg diffraction ("X-ray topography"), reveals isolated defects, such as dislocations, inclusions, stacking faults, and sometimes domain walls, in single crystals. It largely contributed to the development of the processes that now provide the huge perfect crystals of silicon which make microelectronics so efficient.

The advent of synchrotron radiation was a boon to X-ray imaging through the possibilities for real-time observation or for refined beam preparation offered by the increase in intensity. However, the place imaging techniques have taken (15\% of the activity of ESRF, i.e. much more than planned) on third generation sources also results from the entirely new avenues that have been opened through the high geometrical quality of the beams.

In the following, we separate the developments we consider as more closely associated to the gain in intensity (the quantitative aspect) from those more directly connected to the new geometric features (qualitative aspects). The results we describe were obtained on several instruments, mostly but not exclusively at ESRF.

\section{Quantitative progress: absorption imaging ever more refined}

\section{Angiography}

$\mathrm{X}$-ray absorption changes abruptly with the energy or wavelength of the photons at the absorption edges, corresponding to the energy required to eject an electron from a deep atomic level. This variation has been used for the selective observation of blood vessels, in particular for the diagnosis of heart problems associated to the coronary arteries, via angiography. In the hospital version of this technique, a catheter is introduced along an artery to bring a contrast agent (an iodine-containing solution) near the coronary artery to be inspected. This approach involves a fatal risk of about $0.1 \%$. SR makes it possible to obtain excellent images with reduced danger, because an injection of a contrast agent through a vein is enough. The higher dilution is compensated by the logarithmic subtraction of two images. Because the heart moves, the approach consists in simultaneously using two flat beams with slightly different energies, one above, the other below the $\mathrm{K}$ absorption edge of iodine $(33.17 \mathrm{keV})$. The beams cross at the location of the investigated organ, and they are measured by two detector lines, while the patient is translated vertically. Fig. 1 shows the

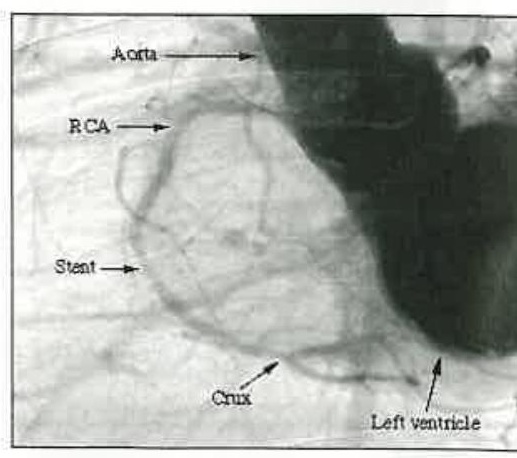

Fig. 1: Image of the heart and arteries o the first human patient at ESRF's Medical Beamline: this differential angiogram results from logarithmic subtraction of two images obtained simultaneously on either side of the iodine absorption edge. The right coronary artery (RCA) shows a stent anc the crux (courtesy H. Elleaume, ESRF [H. Elleaume et al., Phys. Med. Biol. 45, L39L43 (2000)]). 


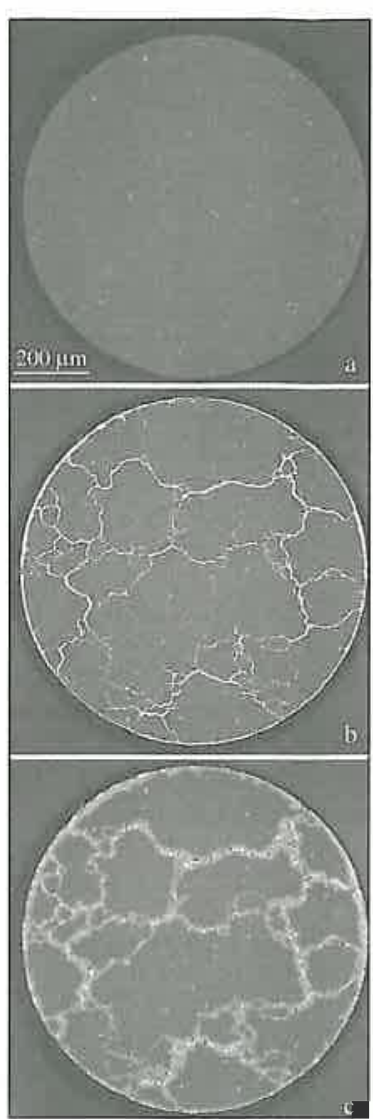

Fig. 2: Study of liquid metal embrittlement by means of high resolution absorption tomography. Reconstructed slices of a polycrystalline aluminium alloy: a) in its initial state b) after exposure for 4 hours to liquid gallium close to room temperature c) after a supplementary anneal for two hours at $300^{\circ} \mathrm{C}$. The gallium appears in b) as white lines at the grain boundaries. These lines become diffuse in c) as gallium diffuses into the aluminium grains. The voxel size in the images is $1 \mu \mathrm{m}$, corresponding to a spatial resolution of $1.7 \mu \mathrm{m}$.

results recently obtained, after preliminary attempts on pigs, on human patients at the medical beamline ID17 of the ESRF.

\section{Imaging through magnetic} dichroism

Refinement is perhaps even more obvious in the use for imaging of magnetic X-ray dichroism, which several groups have developed in the "soft" X-ray range (energy $<1 \mathrm{keV}$ ). In a magnetic material, in the immediate vicinity of an absorption edge, absorption can, for a given polarisation state of the incident beam, depend on the magnetisation state of the sample. It is therefore possible to image the distribution of magnetisation, i.e. the magnetic domains, with only one chemical element at a time being sensed since the energy of the edge is different for each. This is obviously attractive in the case of magnetic multilayers, where the magnetisation directions vary from one layer to the other. Several approaches are used to change the small variation in $\mathrm{X}$-ray absorption into an image. Some use an electronoptical system to produce an enlarged picture through the emitted photoelectrons, which are the more numerous the higher $\mathrm{X}$-ray absorption is. These experiments, based on the use of secondary electrons, and on absorption edges corresponding to soft X-rays, can only be performed in a good vacuum, and only reveal the immediate neighbourhood of the surface. Another approach is the use of a transmission Xray microscope, as at BESSY (Berlin), which proved a powerful tool for probing the magnetisation process in $\mathrm{Gd}-\mathrm{Fe}$ multilayers.

\section{Absorption microtomography}

The three-dimensional (3D) reconstruction of complex structures, on the basis of many images obtained for different orientations of the sample with respect to the beam, is possible with spatial resolution down to $1 \mu \mathrm{m}$ (microtomography). This is the smaller-scale analogue of the medical scanner or CAT. From the tomographic reconstruction, one can at will produce cuts or volume renderings of the object. The number of $2 \mathrm{D}$ images required is approximately equal to the number of pixel columns in the detector. At the imaging beamline ID19, this number is typically 900 when using a $1024 \times 1024$ pixel CCD camera. The time required for recording these $2 \mathrm{D}$ images with an extended, parallel and moderately monochromatic $\left(\triangle E / E \approx 10^{-2}\right)$ beam is now less than 10 minutes, while the $3 \mathrm{D}$ reconstruction time, on a powerful computer, remains on the order of one hour. The spatial resolution is mainly determined by the detector specially developed for these experiments. The usual tool is an X-ray --> visible light converter screen coupled, via visible light optics, to a cooled CCD camera (FRELON, for Fast REad-out, LOw Noise) developed at ESRF.

Under specific conditions a liquid metal can penetrate into the grain boundaries of a polycrystalline solid metal, leading to a brittle behaviour of the normally ductile solid. This phenomenon, known as liquid metal embrittlement, was discovered more than a century ago. However, the mechanisms leading to rapid penetration along grain boundaries remain poorly understood. Absorption radiography and tomography, with a spatial resolution of about 1 micron, are well suited to studies on the kinetics of this process in the case of aluminium alloys. Gallium, which is liquid just above room temperature, attenuates $\mathrm{X}$-rays much more than aluminium. This makes it possible to observe in-situ the penetration of gallium into the bulk. Figure 2 shows the same virtual slice of an aluminium alloy ( $\mathrm{Al} \mathrm{5038)} \mathrm{at} \mathrm{various} \mathrm{stages.} \mathrm{The} \mathrm{first}$ tomographic image (figure 2a) shows the initial state of the sample; the isolated white points correspond to $\mathrm{Fe}$ and $\mathrm{Mn}$ rich inclusions. Figure $2 \mathrm{~b}$ was recorded after liquid gallium was allowed to penetrate, and after annealing the sample close to room temperature: the white lines that divide the sample into cells indi-
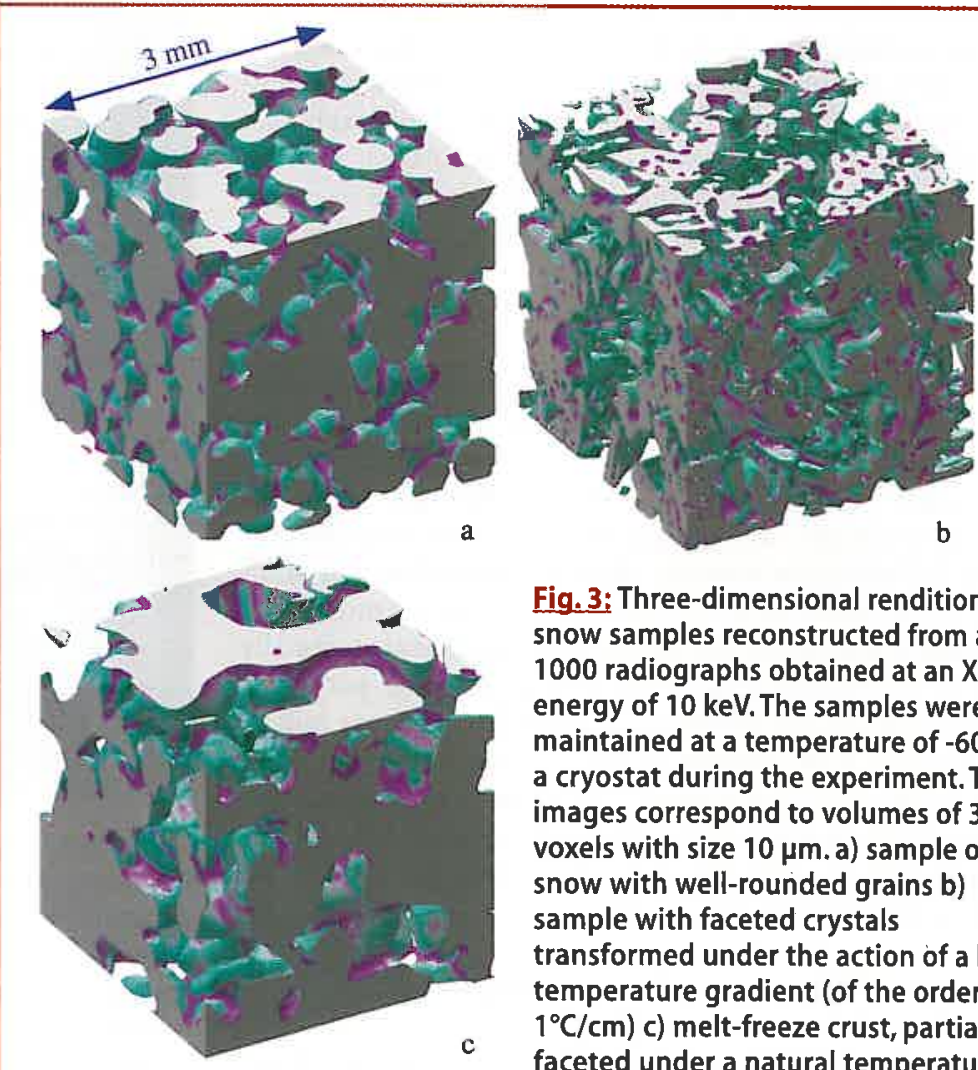

Fig. 3: Three-dimensional renditions of snow samples reconstructed from about 1000 radiographs obtained at an X-ray energy of $10 \mathrm{keV}$. The samples were maintained at a temperature of $-60^{\circ} \mathrm{C}$ in a cryostat during the experiment. The images correspond to volumes of $300^{3}$ voxels with size $10 \mu \mathrm{m}$. a) sample of wet snow with well-rounded grains b) sample with faceted crystals transformed under the action of a large temperature gradient (of the order of $1^{\circ} \mathrm{C} / \mathrm{cm}$ ) c) melt-freeze crust, partially faceted under a natural temperature gradient (courtesy C. Coléou). 
cate the presence of gallium along the grain boundaries. The lines become diffuse and heterogeneous after a second anneal which allows gallium to diffuse into the grains (figure $2 \mathrm{c}$ ): isolated gallium precipitates and cavities can be observed. These experimental observations should corroborate some of the antagonistic models proposed in the literature.

Figure 3 is a $3 \mathrm{D}$ rendition of spring snow that suffered metamorphic transformations. Metamorphism has radical consequences: the physical and mechanical properties can change over several orders of magnitude. This is related to the fact that some snows stick to almost vertical rock walls, while others yield under the weight of a single skier. The form and arrangement of the grains, and the quality of the bonds in the ice, are key factors in determining the properties of snow. The growth of ice particles is associated with the diffusion of vapour in dry snow, and to melting / resolidification in damp snow. Dry snows are usually warmer at the bottom. The value of the temperature gradient determines whether the crystallites take on a rounded (small gradient) or facetted (high gradient) form. The important parameters are the area per unit volume, the inter-grain connections, and the local radius of curvature of the grains. These parameters cannot be directly measured on $2 \mathrm{D}$ images. 3D images such as those of fig. 3 provide important data on statistically significant volumes, containing enough grains, and with spatial resolution limit much smaller than the grain size. They are quantitatively evaluated by a research group at the Snow Research Centre (Centre d'Etudes de la Neige) of the French weather agency (Météo-France).

These examples of synchrotron radiation absorption microradiography show two trends : the use of high spatial resolution in the first case, and specially designed sample environments in the second. Quantitative tomography, made possible by the monochromaticity of the X-ray beam, shifts the emphasis from the sample architecture, i.e. the geometry, to the densitometry of the solid parts through mapping of the linear absorption coefficient. Another development is "local" or "zoom" tomography, the high resolution reconstruction of a region of interest within a matrix that gets only low resolution reconstruction. This is essential for applications where it is not possible or not desirable to extract a small sample from its matrix.

\section{Zone plate microscopes}

The advent of synchrotron radiation also stimulated the development of microscopes for soft X-rays, in particular for the wavelength range (the "water window") where absorption by carbon is much stronger than by water. They are based on make-shifts for lenses, zone-plates, and reach very valuable performance for biological systems. Thus, 3D reconstruction of an alga cell was achieved, with a spatial resolution on the order of 60 $\mathrm{nm}$. The use of zone plates also allows refinements that are usually thought to be possible only in the visible range, in particular Zernike's phase contrast technique. A transmission X-ray microscope operating with energies in the 2-7 keV range has been installed on the ID21 beamline of ESRF. The possibility to tune the energy to the vicinity of important absorption edges $(\mathrm{Ca}, \mathrm{Cr}$, ...) allows different elements and their chemical state to be mapped through spectro-microscopy.

\section{Qualitative progress: scanning X-ray imaging, phase radiography and phase tomography}

Two new families of imaging techniques depend more directly on the beam quality reached in third generation synchrotron radiation. They are the scanning imaging techniques on the one hand,
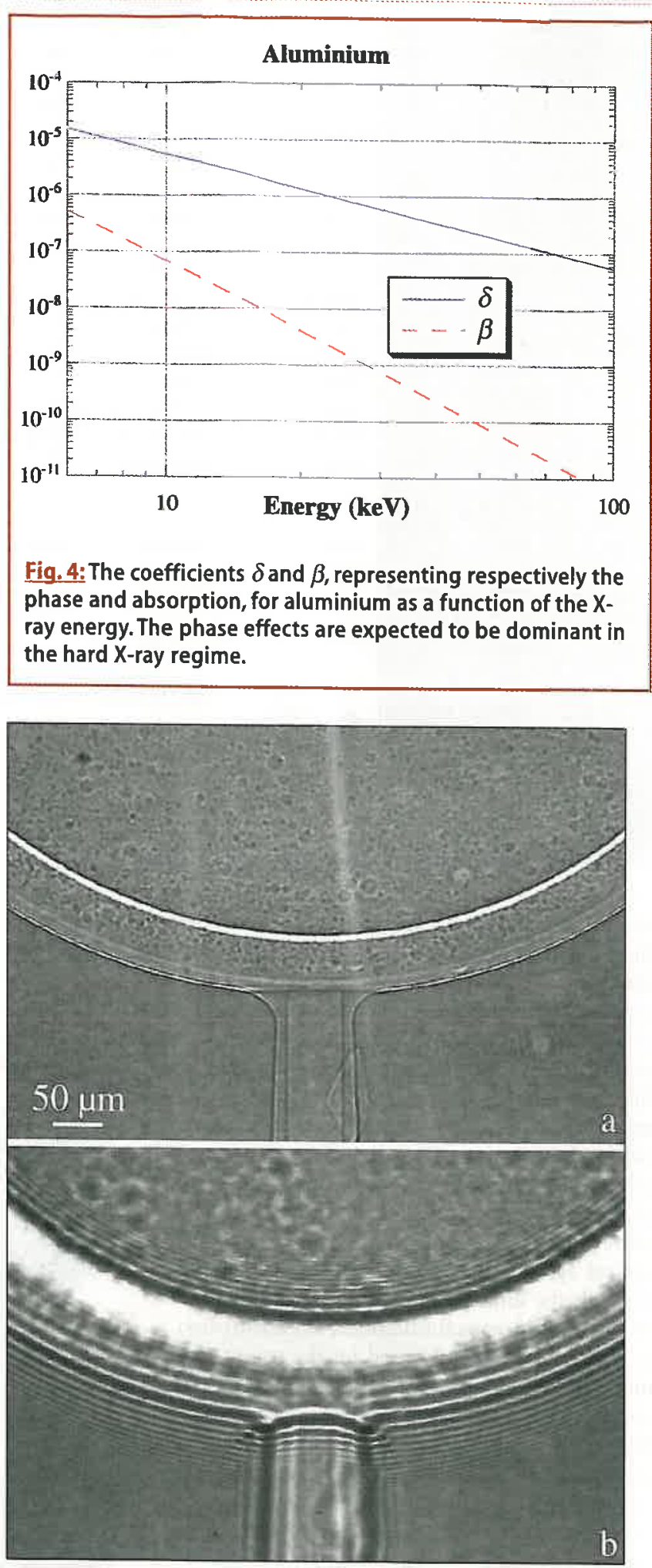

Fig. 5: Two images of a hollow sphere consisting of two different polymer layers. In image a), recorded at a sampledetector distance of $19 \mathrm{~cm}$ and representative of the edgedetection regime, the borders are revealed by white-black contrast. In image b), recorded at a distance of 3 metres and representative of the holographic regime, many interference fringes are observed. 
and the easy observation of inhomogeneities in the optical phase of the outgoing beam on the other hand.

\section{$X$-ray scanning imaging}

The X-ray beams produced by undulators at third generation SR sources can, because they originate in a very small source, be focused into a very fine spot (on the order of a micrometer in linear size) with extremely high intensity. It is then possible to scan the sample with respect to the beam, with an appropriate detector or bank of detectors collecting quantitative information. This information is then displayed on an electronic device as a point with variable intensity and colour at the coordinates corresponding to the position of the beam. This provides the exact analogue of scanning electron microscopy. There is a wide variety in the type of information available : wide angle diffracted X-rays, or small-angle scattered X-rays, fluorescence photons, or secondary electrons. A whole range of scanning $\mathrm{X}$-ray imaging techniques appeared, and they will certainly develop brilliantly over the next few years.

\section{Why phase?}

A beam of X-rays going through an object suffers; apart from absorption, a phase shift, because the refractive index of materials is slightly different from unity. The complex refractive index $\mathrm{n}=1-\delta+\mathrm{i} \beta$ describes both absorption (related to $\beta$ ) and the phase shift with respect to the beam in vacuum:

$$
\varphi(x, y)=-(2 \pi / \lambda) \int \delta(x, y, z) \mathrm{d} z
$$

where the integral is taken along the path of the beam. This phase is non-uniform over a cross-section of the X-ray beam if the thickness and/or the structure of the sample are inhomogeneous. $\delta$ is proportional to the electronic density of the material, hence approximately to its mass density; it must be corrected for dispersion when the photon energy is near an absorption edge. The phase cannot be measured directly, and variations in phase do not affect the intensity of the beam as it exits the sample. Phase images have been obtained, using laboratory sources or second generation synchrotron radiation, using elaborate devices (interferometers, many-crystal setups). With many images of the sample recorded while it is rotated in an interferometer, it is possible to reconstruct in $3 \mathrm{D}$ the distribution of refractive index, i.e. to perform phase tomography.

The qualitatively new character of third generation SR is that its lateral coherence makes it possible to visualise and reconstruct the phase variations of hard X-rays, both in simple radiography and in tomography, with great instrumental simplicity. Furthermore, better spatial resolution can be reached compared to existing phase imaging techniques. The required coherence is obtained thanks to the very small angular size $\alpha$ of the source as seen by a point in the sample (less than a microradian on the imaging beamline ID19), which entails a large lateral coherence $L_{c}$ of the X-ray beam, with wavelength $\lambda$ :

$$
L_{\mathrm{c}}=\lambda / 2 \alpha
$$

For example, $L_{\mathrm{c}} \approx 100 \mu \mathrm{m}$ for $\lambda \approx 1 \AA$. In optical terms, the effect used to turn local variations of phase into intensity variations is interference, at finite distance, between parts of the beam that have suffered different phase shifts but are coherent with one another. This is the analogue of defocusing in electron microscopy: it can be described in terms of Fresnel diffraction, or of in-line holography.
In practice, obtaining a phase-sensitive image just involves setting the detector (film or CCD camera) at a distance $D$ on the order of one tenth of a meter from the sample. An absorption image is obtained if $D$ is small ( $\mathrm{mm}$ range). This corresponds to the fact that the region in the sample that affects the image at a point of the detector has a size $r=\sqrt{ }(\lambda D)$, the radius of the first Fresnel zone. When $D$ is a few $\mathrm{mm}$, the size of this zone is below the resolution of the detector $(\mu \mathrm{m})$, and no interference will be observed: only absorption contrast will be effective. For larger values of $D$, but with $r$ remaining small with respect to the size $a$ of the object to be imaged, the edges of the object behave independently, and are the only contribution to the image. The best sensitivity to a phase object of size $a$ is obtained for a distance $D \approx$ $a^{2} / 2 \lambda$, but then the image is a hologram which does not look much like the object.

The major advantage of this new type of imaging is the increased sensitivity it provides. Fig. 4 shows, for aluminium, that $\delta>>\beta$ in the hard X-ray range $(E>6 \mathrm{keV})$. This explains the gain observed, particularly for light materials such as polymers, or for composites consisting of materials which equally attenuate $\mathrm{X}$-rays, such as $\mathrm{Al}$ and $\mathrm{SiC}$.

The phase variations across the beam at the sample exit lead to variations in intensity, hence to contrast, provided the phase has a non-vanishing two-dimensional Laplacian, $\left(\partial^{2} \varphi / \partial x^{2}\right)+\left(\partial^{2} \varphi / \partial y^{2}\right) \neq 0$. They show up, for increasing values of $D$, through the appearance of a black-white line at the phase

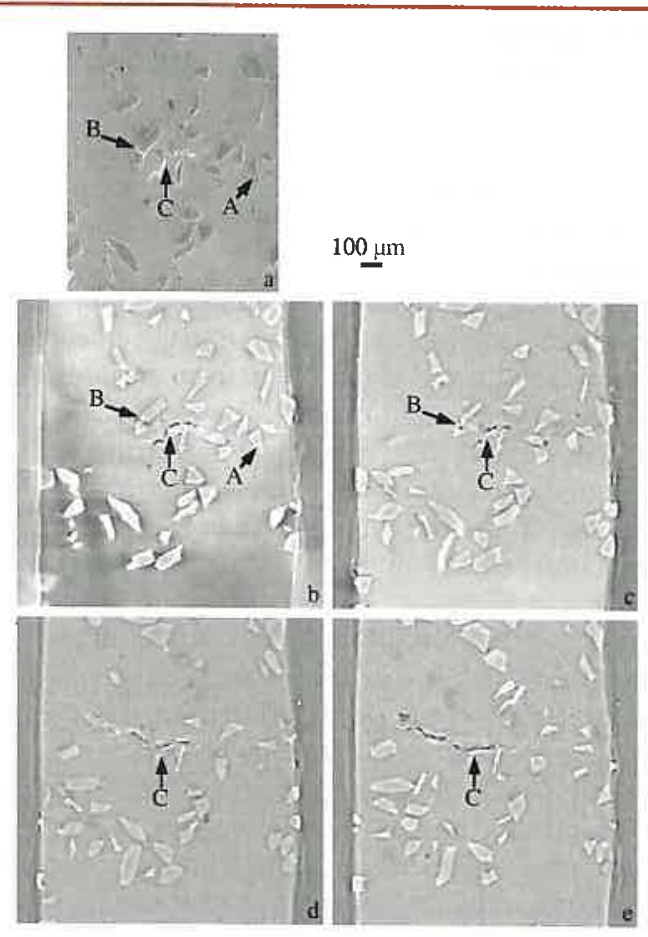

Fig. 6: a) Scanning electron microscope image of the surface of a composite material consisting of an aluminium matrix reinforced by $\mathrm{SiC}$ particles $b$ )-e) Slices obtained by computed tomography corresponding to zones respectively at the surface, and $25 \mu \mathrm{m}, 50 \mu \mathrm{m}$ and $75 \mu \mathrm{m}$ below the surface. The sample suffered a monotonic tensile test of $1 \%$. Cracked SiC particles can be detected (arrows A and $B$ ), as well as a crack in the aluminium matrix (arrow $C$ ). The tomographic acquisition was performed at an X-ray energy of $25 \mathrm{keV}$ and a sample-detector distance of $0.82 \mathrm{~m}$ (collaboration with J.-Y. Buffière, INSA, Lyon). 


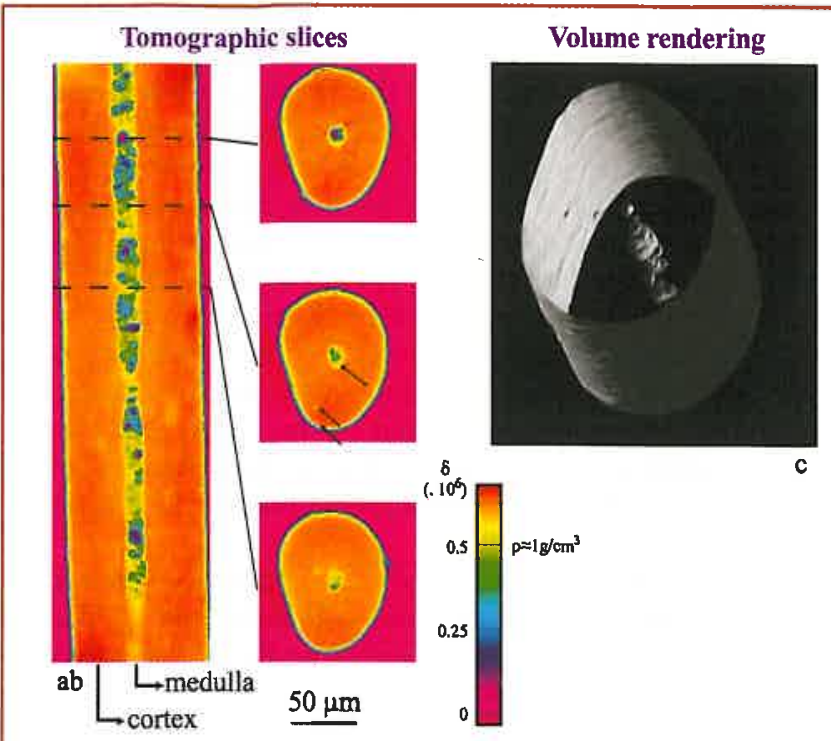

Fig. 7: Results of 3D density mapping (holotomography) of human hair. The tomographic slices in the a) axial and b) radial direction show the medulla as a low density region within the cortex. Cavities exist along the medulla and the arrows indicate low density regions. c) 3D rendition of the hair revealing the medulla within the outer surface. The 3D density mapping involves a phase retrieval method based on images recorded at several distances (three in this case), a procedure that is repeated for a large number of angular positions (X-ray energy $21 \mathrm{keV}$ ).

jumps (fig. 5a), then by a set of Fresnel interference fringes which become more and more obtrusive (fig. $5 b$ ): the image is then an in-line hologram.

The set-up used is simple: on the imaging beamline ID19 at ESRF, it essentially consists in a monochromator made up of perfect silicon crystals, located about $140 \mathrm{~m}$ from the insertion device (a variable gap wiggler or an undulator) which acts as its photon source. Of course, phase inhomogeneities give an image also if they do not arise from the sample. To avoid these unwanted images, the optical elements in the beamline (beryllium windows, filters and monochromators) are submitted to unusually stringent demands.

\section{Tomographic observation through edge-enhancement}

The next step consisted in extending this possibility of observing phase objects to three dimensions, in other words in going over to phase tomography. It was performed in two steps.

It turns out that the tomographic reconstruction codes developed for absorption images yield acceptable results when the phase images result from density jumps with sharp edges. In metallurgy, $\mathrm{X}$-ray phase microtomography is particularly valuable for systems involving regions with neighbouring compositions. As shown by fig. 6 , it has provided important results for the understanding of degradation mechanisms in aluminium-SiC composites. In this case, it is possible both to visualise the $\mathrm{SiC}$ particles easily, and to observe in situ the nucleation and growth of cracks when the sample is submitted to traction. These cracks occur first in the elongated particles, and this imaging technique shows that there are $50 \%$ more of these cracks than indicated by investigations restricted to the surface. The experimental results are now incorporated in programs that model the mechanical behaviour of these composite materials.

\section{Quantitative reconstruction of the phase map}

The second, recent step consisted in developing a procedure for the "holographic" reconstruction of the phase maps, which can serve as the more correct input data for the "tomographic" reconstruction. Phase retrieval is based on the use of a few images recorded at different distances from the sample. It therefore leads to acquisition times longer than for absorption tomography. The approach is derived from a method originally developed for electron microscopy by a group in Antwerp. It is quantitative, and determines the phase, well beyond simple edge images, with a spatial resolution limited by the detector (about 1 $\mu \mathrm{m}$ in our case). The combination with tomographic reconstruction, called holotomography, yields 3D quantitative images that show the distribution of electron density, hence of mass density, in the sample.

Figure 7 shows an example of 3D density mapping obtained on a human hair. The axial slice (figure 7a) through the middle of the hair shows that the medulla, the central hair region, is very inhomogeneous and varies along the hair shaft. The density is in general lower than the surrounding cortex with some regions revealed as cavities. The radial slices (figure7b) show low density regions, at the level of the medulla, but also near the outer border of the hair and in particular points of the cortex. The lower density of some regions can be ascribed to a higher lipid concentration and lower protein content.

\section{Conclusion}

The advent of third generation synchrotron radiation sources such as ESRF has opened new possibilities in X-ray imaging. These techniques are now beyond the stage of demonstration experiments, and they are appreciated in the solution of various scientific problems in materials science, biology and medicine. The applications are based, as far as absorption imaging is concerned, on the very broad choice in photon energy available (typically between 1 and $120 \mathrm{keV}$ ), which makes it possible to improve the contrast, on the improved spatial resolution (on the order of $\mathrm{a} \mu \mathrm{m}$ ), and on the quantitative data evaluation made possible by the monochromatic and parallel character of the beam. Furthermore, the very small source size makes scanning imaging techniques possible. It also provides, in an instrumentally simple way, phase images which reveal phenomena that are difficult to evidence by other means. This new technique can be used for twoor three-dimensional imaging, either qualitatively for edge detection (pores, inclusions...), or quantitatively, through phase retrieval complemented by tomographic reconstruction, in the approach called holotomography.

\section{References}

[1] Developments in X-Ray Tomography II Ulrich Bonse, Editor

Proceedings of SPIE Vol. 3772 (1999).

[3] Instrumental aspects of $x$-ray microbeams in the range above $1 \mathrm{keV}$ Dhez P., Chevallier P., Lucatorto T.B., Tarrio C. Rev. Sci. Instrum. 70, 1907-1920 (1999).

[2] Hard X-ray phase imaging using simple propagation of a coherent synchrotron radiation beam

Cloetens P., Ludwig W., Baruchel J., Guigay J.P., Rejmànkovà-Pernot P., Salome-Pateyron M., Schlenker M., Buffiere J.Y., Maire E., Peix G. J. Phys. D: Appl. Phys. 32, A145-A151 (1999).

[4] Holotomography: Quantitative phase tomography with micrometer resolution using hard synchrotron radiation $\mathrm{X}$-rays

Cloetens P., Ludwig W., Baruchel J., Van Dyck D., Van Landuyt J. , Guigay J.P., Schlenker M.

Appl. Phys. Lett., 75, 2912-2914 (1999). 